\title{
Red-Emitting $\Pi$-Conjugated Oligomers Infused Single-Wall Carbon Nanotube Sheets
}

\author{
Toshihiko Fujimoria,b,* and Koki Urita ${ }^{\mathrm{c}}$ \\ ${ }^{a}$ Center for Energy and Environmental Science, Shinshu University, 4-17-1 Wakasato, \\ Nagano-city 380-8553, Japan \\ bJST, PRESTO \\ cDivision of Chemistry and Materials Science, Graduate School of Engineering, Nagasaki \\ University, 1-14 Bunkyo-machi, Nagasaki-shi, Nagasaki 852-8521, Japan \\ ${ }^{*}$ Corresponding author: T. Fujimori \\ e-mail:t_fujimori@shinshu-u.ac.jp \\ Tel: +81-26-269-5758 \\ Address: 4-17-1 Wakasato, Nagano-city 380-8553, Japan
}

\section{Keywords:}

single-wall carbon nanotubes, $\Pi^{-c o n j u g a t e d ~ o l i g o m e r s, ~ n a n o h y b r i d ~ m a t e r i a l s, ~}$ fluorescence

\section{Abstract}

We demonstrate the one-step thermal fusion and infusion of pyrene molecules inside single-wall carbon nanotubes (SWCNTs). Despite the presence of metallic-SWCNTs, which behave as a quencher due to gapless electronic states, the nanohybrids consisting of pyrene and/or azupyrene oligomers infused SWCNT sheets exhibit red fluorescence by the ultraviolet, blue, and green light excitations. The wavelength-independent lightemitting behaviour is explained by (1) infused PAH oligomers inside semiconductingSWCNTs and (2) the peculiar $\Pi^{-} \Pi$ interaction through mixed $\Pi^{-}$conjugated state between

(c) 2016. This manuscript version is made available under the Elsevier user license http://www.elsevier.com/open-access/userlicense/1.0/ 
the $\Pi^{-}$conjugated oligomers and non-armchair metallic-SWCNTs.

\section{Introduction}

The unique optical property of single-wall carbon nanotubes (SWCNTs) has widened those potential applications into next-generation optoelectronic devices [1-3], electrochromic devices [4], chemical sensors [5,6], and biological sensors [7,8]. Due to the narrow band gap of semiconducting-SWCNTs, the light-emitting property of SWCNTs are, in principle, limited to the near-infrared region [9,10]. In addition, the presence of gapless metallic-SWCNTs acts as a quencher, so that it is needed to prevent the intertube interactions with metallic-SWCNTs for photo-emissions. Therefore, SWCNTs show photoluminescence in only liquid suspensions and/or semiconducting-enriched SWCNT sheets [11]. Since as-produced SWCNTs consist of both metallic- and semiconducting SWCNTs, a key challenge is to further extend the light-emitting property of SWCNT sheets into the visible-light range without separation of metallic-SWCNTs.

The peculiar ability of SWCNTs is to stabilize exotic molecules into their onedimensional nanochannels [12-15]. To date, few flourescent molecules are known to exhibit photoluminescence inside SWCNTs. Clear examples are found in 1D-stacked coronene molecules [16], graphene nanoribbons [17], and $\alpha^{-}$sexithiophene [18] infused 
SWCNTs in liquid suspensions. In order to emit light by a sheet consisting of both metallic- and semiconducting-SWCNTs, our strategy is to form a mixed $\Pi^{-}$-conjugated state between metallic-SWCNTs and infused small polycyclic aromatic hydrocarbons (PAHs) of which emission wavelengths can be in principle tuned for closely matching to the narrowest gap energy of metallic-SWCNTs by extending $\Pi^{-c o n j u g a t e d ~ l e n g t h s ~}$ through thermal fusion reactions [19,20].

\section{Methods}

\subsection{Experimental}

Reagents were obtained from commercial suppliers and used without further purification. We used SWCNTs produced by the arc-discharged method (ASP-100F, Hanwha Nanotech). Average diameter of the SWCNT sample was $1.5 \mathrm{~nm}$. The SWCNT sample was not processed to any separation methods of metallic- and semiconductingSWCNTs, thus the sample includes both metallic- and semiconducting-SWCNTs. Prior to infuse PAHs, the SWCNT sample was oxidized by heating at $723 \mathrm{~K}$ under dry air (100 $\mathrm{ml} \mathrm{min}-1$ ) for removing the terminating caps. The opened SWCNTs and pyrene (purity: $98.0 \%)$ with weight ratio of 1:20 were sealed in a glass tube in vacuo $(<1 \mathrm{~Pa})$, and subsequently kept at $773 \mathrm{~K}$ for $48 \mathrm{~h}$. To remove the excess pyrene and/or azupyrene 
attached to the outside of the SWCNTs, the as-synthesized sample was then extensively washed by toluene under ultrasonication until color of the solution became transparent. The resulting material was a sheet form and hereafter referred to as PAH@SWCNTs. We also performed annealing of bulk pyrene for comparison. Pyrene was sealed in a glass tube in vacuo $(<1 \mathrm{~Pa})$, and then heated at $773 \mathrm{~K}$ for $48 \mathrm{~h}$.

\subsection{Analytical techniques}

High-resolution transmission electron microscopy (HRTEM) observations were carried out with a HR scanning transmission electron microscope (ARM200CF, JEOL) operated at $80 \mathrm{kV}$. Optical absorption spectra were obtained using a UV-VIS-NIR spectrometer (V670, JASCO). Mass spectra were obtained by the electron ionization mode using a thermogravimetric (TG) analysis-mass spectrometer (ThermoMASS, Rigaku). TG-mass measurements were carried out under He gas flow $\left(300 \mathrm{ml} \mathrm{min}^{-1}\right)$ in the temperature range $300-1273 \mathrm{~K}$ at a scan rate of $5 \mathrm{~K} \mathrm{~min}^{-1}$. X-ray diffraction (XRD) were measured using the synchrotron X-ray source at SPring-8 $(\lambda=0.09982 \mathrm{~nm})$. Raman spectra were obtained with 488, 532, 633, and $785 \mathrm{~nm}$ excitations using a single-monochromator Raman spectrometer (T64000, HORIBA Scientific). Fluorescent microscopic images were acquired using an optical microscope (BX51, OLYMPUS) with mercury lamp as an excitation light source. 


\section{Results and Discussion}

Figure 1(a) shows a representative HRTEM image of PAH@SWCNT, indicating the successful infusion of PAH oligomers inside SWCNTs. We can identify two parallel PAH oligomers preferentially aligned along the inner wall of an SWCNT, resulting from the interaction potentials by the surrounding SWCNT walls. Since our HRTEM analysis reveals that the average $\mathrm{PAH}$ oligomers-tube spacing is $0.36 \mathrm{~nm}$, we can expect the specific $\Pi^{-} \Pi$ interaction between PAH oligomers and SWCNTs rather than that between PAH oligomers.

The observed HRTEM image of infused PAH oligomers consists of two different structures: (1) straight linear lines (highlighted in the red region in Fig. 1(a)) and (2) corrugated lines (highlighted in the green region). In order to identify those different structural images of infused PAH oligomers, we performed TEM image simulations of pyrene-fused oligomers and azupyrene-fused oligomers inside SWCNTs. Azupyrene (dicyclopenta[ef,kl]heptalene), which is a non-alternant isomer of pyrene, consists of two adjacent pentagon-heptagon backbones of carbon atoms [21]. Thermal isomerization of pyrene to azupyrene could be occurred through the thermal isomerization process [22]. Fig. 1(b) shows HRTEM simulated images of azupyrene oligomers inside a metallic $(9,9)$ 
SWCNT. The model structures of azupyrene oligomers inside $(9,9)$ SWCNT are depicted in Fig. 1(c). Here two possible molecular plane configurations of infused azupyrene oligomers are considered, such as perpendicular (model structure shown in the left side of Fig.1 (c)) and parallel (shown in the right side of Fig. 1(c)) molecular planes to the incident electron beam. Compared with the observed HRTEM image and the simulated one, the observed straight linear lines inside a SWCNT (highlighted in the red region in Fig. 1(a)) are well reproduced by the simulation images of the parallel configuration of azupyrene oligomers (highlighted by the red region in Fig. 1(b)) and/or pyrene oligomers (not shown). Note that both pyrene and azupyrene oligomers show very similar simulated images if the molecular planes are set parallel to the incident electron beam.

The observed corrugated lines (highlighted in the green region in Fig. 1(a)) can be assigned to infused azupyrene oligomers, which molecular plane is set on the perpendicular configuration. This interpretation is further rationalized by TEM simulation analysis using infused pyrene and azupyrene oligomers on the perpendicular configurations (Fig. 1(d) and (e)). Here we used (11,7) SWCNT as another model for image simulations because its chirality is different from $(9,9)$ SWCNT but its diameter is almost identical to $(9,9)$ SWCNT. The corrugated image is clearly reproduced in the simulated image of the infused azupyrene oligomers inside (11, 7) SWCNT, implying 
that the observed corrugated image in Fig. 1(a) results from the perpendicular configuration of azupyrene oligomers.

To check whether thermal isomerization and fusion of pyrene molecules occurs only inside SWCNTs, pyrene was annealed at $773 \mathrm{~K}$ without the presence of SWCNTs. In contrast to pristine pyrene which is a yellow crystal, the annealed pyrene was obtained as a reddish brown powder of which color is very similar to that of azupyrene synthesized by the organic synthesis method [21]. The plausible formation of azupyrene through the annealing process is rationalized by optical absorption analysis shown in Fig. 2. Fig. 2 shows optical absorption spectra of $2 \times 10^{-5} \mathrm{M}$ of thermally-treated pyrene and pyrene in toluene solutions. In addition to the sharp absorption bands at 300-350 $\mathrm{nm}$, thermallytreated pyrene exhibits weak bands at $416,433,443,458,484,491,518,557$, and 617 nm (Fig. 2(b)). Those absorption wavelengths qualitatively show good accordance with those obtained by the synthesized azupyrene [21].

Figure 3 shows TG-mass spectra of pyrene and thermally-treated pyrene. In addition to the main peak at $m / z=202$, pyrene shows some fragment peaks at $m / z=201,200$, 199, 175, 174, 150,102,101, 100,99, 88, 87, 75, 74, and 63 [23]. On the other hand, thermally-treated pyrene shows a strong peak at $\mathrm{m} / \mathrm{z}=202$ and only two fragment peaks at $m / z=201$ and 178 . The peaks at $m / z=202$ and 201 originate from the original 
molecular ion, $\left[\mathrm{C}_{16} \mathrm{H}_{10}\right]^{+}$, and $\left[\mathrm{C}_{16} \mathrm{H}_{9}\right]^{+}$, respectively, indicating that the resulting material is an isomer of pyrene $\left(\mathrm{C}_{16} \mathrm{H}_{10}\right)$. We interpret the weak peak at $m / z=178$, which is characteristic to thermally-treated pyrene, as a consequence of fragmentation of two carbon atoms from the original molecule. We deduce from the analogous structural conformation between azupyrene and the so-called Stone-Wales defect (two pentagonal and heptagonal rings of carbon atoms) in the hexagonal lattice of graphene [24] that two carbon atoms centered at azupyrene molecule could be knocked off by the electron ionization process of mass spectrometry measurements, resulting in the fragment peak at $m / z=178\left(\left[\mathrm{C}_{14} \mathrm{H}_{10}\right]^{+}\right)$in the mass spectrum. The TG-mass analysis also indicates that it is unlikely to fuse pyrene molecules without SWCNTs by the annealing treatment at $773 \mathrm{~K}$. Although the thermal isomerization of pyrene to azupyrene occurs regardless of the presence of SWCNTs, "nano-channels" of SWCNTs are essential for pyrene and/or azupyrene to form PAH oligomers through the thermal process used in this study. This "nano-channel" induced oligomerization of small PAH molecules is consistent with the previous report by Talyzin et al. [20].

Figure 4 shows XRD profiles of empty SWCNTs and PAH@SWCNTs. Empty SWCNTs exhibit characteristic Bragg peaks which are assigned to the two-dimensional triangular lattice of SWCNT bundles [25]. The most important XRD feature of infused SWCNTs is 
that intensity of the (10) peak decreases by filling exotic molecules inside the hollow cores of SWCNTs (e.g., filling $\mathrm{C}_{60}[26]$ and dye molecules [14]). Here the XRD profile of PAH@SWCNTs clearly shows the significant drop of the (10) peak intensity, indicating the successful infusion of pyrene and/or azupyrene oligomers inside the SWCNTs. In addition, the XRD profile of PAH@SWCNTs shows a very broad peak at the scattering vector $Q \sim 17 \mathrm{~nm}^{-1}(d$-spacing $=0.34 \mathrm{~nm})$. This $d$ value is very close to the PAH-tube distance evaluated by the HRTEM analysis $(0.36 \mathrm{~nm})$. Not only decrease of the (10) peak intensity, but also the presence of this broad Bragg peak strengthens the evidence of highly-ordered infusion of the PAH oligomers inside the SWCNTs in large quantity.

In addition to the optical transition bands of empty SWCNTs, which are assigned to the first electronic transitions of metallic-SWCNTs $\left(\mathrm{M}_{11}: 1.5-2.0 \mathrm{eV}\right)$ and the second and third electronic transitions of semiconducting-SWCNTs $\left(\mathrm{S}_{22}: 1.1-1.4 \mathrm{eV}\right.$ and $\mathrm{S}_{33}$ : $2.0-2.5 \mathrm{eV}$, respectively) [27,28], PAH@SWCNTs in $1 \mathrm{wt} \%$ aqueous sodium dodecyl sulfate (SDS) solution show broad absorption bands in the wavelength region between $450-800 \mathrm{~nm}$ (the bottom panel in Fig. 5(a)). Here the upper panel in Fig. 5(a) indicates absorption bands of infused PAH oligomers, which are extracted by subtracting the optical absorption spectrum of empty SWCNT from that of PAH@SWCNT. In order to provide further insight into the broad optical bands observed in PAH@SWCNTs, we 
performed ab initio time-dependent density functional theory (TD-DFT) calculations at the B3LYP/6-31G(d) level using the GAUSSIAN 09 code [29] for pyrene, azyupyrene and those oligomers. Fig. 5(b) shows the TD-DFT calculated optical absorption spectra of the pyrene and azupyrene oligomers $(n=1-4, n$ : a number of fused monomers) obtained after calculations of the optimized geometries. As $n$ increases, the optical absorption bands of both pyrene and azupyrene oligomers shift to longer wavelengths and the bands become broader. Compared with the same $n$ value of pyrene and azupyrene oligomers, the optical absorption bands of azupyrene oligomers tend to show higher wavelength than those of pyrene oligomers. Although it is difficult to determine the difference between pyrene and azupyrene oligomers in the observed optical spectra, the broad and featureless optical band of infused PAH molecules shown in Fig. 5(a) provides evidence of the formation of pyrene and/or azupyrene oligomers $(n=2-4)$ inside SWCNTs.

Figure 6(a) shows resonance Raman spectra of empty SWCNT and PAH@SWCNT sheets recorded with the 488, 532, 633, and $785 \mathrm{~nm}$ laser excitations. Raman spectra of thermally-treated pyrene recorded with the 532 and $633 \mathrm{~nm}$ laser excitations are shown in Fig. 6(b) for comparison. In addition to the Raman modes of SWCNTs, which are assigned to the G-modes ( 1590 $\left.\mathrm{cm}^{-1}\right)$ and the radial-breathing modes (150-170 $\left.\mathrm{cm}^{-1}\right)$ [30], PAH@SWCNT exhibits characteristic Raman bands at 1178, 1202, 1240, 1281, 1366, 
1390, 1526, and $1571 \mathrm{~cm}^{-1}$ (arrows in Fig. 6(a)) and some weak spectral features below $1000 \mathrm{~cm}^{-1}$. Obviously, those Raman bands, which we assign to vibration modes of infused PAH oligomers, are significantly enhanced by the 633 and $785 \mathrm{~nm}$ excitations. On the other hand, the Raman bands of infused PAH oligomers become less pronounced with the 488 and $532 \mathrm{~nm}$ excitations. According to the Kataura plot [27], the metallicSWCNTs are in resonance with the 633 and $785 \mathrm{~nm}$ excitations due to the $\mathrm{M}_{11}$ transitions (Fig. 5(a)). Interestingly, infused PAH oligomers shows the optical absorption bands at the same wavelength region (Fig. 5(a)), implying that co-excitations of metallic-SWCNTs and PAH oligomers play an important role for the strong Raman enhancement. We could rule out the resonance effect simply due to the excitation of infused PAH oligomers, since the Raman enhancement is obscured with the $532 \mathrm{~nm}$ excitation. The observed Raman enhancement could be interpreted as the so-called surface-enhanced Raman scattering due to the charge transfer mechanism [31]. It is known that $s p^{2}$ carbon materials (e.g., graphite) can adsorb many organic molecules on the surfaces, exhibiting strong Raman enhancements due to the charge transfer mechanism. For example, photo-excited rhodamine 6G molecules transfer its energy to the conducting carbon materials and show the Raman enhancement [32]. More recently, much efforts have been devoted using graphene as a Raman enhancer, called "Graphene-enhanced Raman scattering [33]". 
Based on those prior works using $s p^{2}$ carbon materials, we expect that the observed Raman enhancement originates from a specific interaction between photo-excited metallic-SWCNTs and infused PAH oligomers.

Figures 7(a-c) shows fluorescent microscopic images of PAH@SWCNT sheets excited with the ultraviolet $\left(\lambda_{\mathrm{ex}}=360-370 \mathrm{~nm}\right)$, blue $\left(\lambda_{\mathrm{ex}}=470-495 \mathrm{~nm}\right)$, and green $\left(\lambda_{\mathrm{ex}}=530\right.$ $-550 \mathrm{~nm}$ ) light sources. For comparison, fluorescent microscopic images of thermallytreated pyrene (azupyrene) are shown in Figs. $7(d-f)$. Here, the detection wavelengths are $>420 \mathrm{~nm},>510 \mathrm{~nm}$, and $>575 \mathrm{~nm}$ for the ultraviolet, blue, and green light excitations, respectively. Despite the presence of metallic-SWCNTs, which are believed to act as quenchers, PAH@SWCNT sheets exhibit the red emission and the fluorescent color is likely independent of the excitation wavelengths. In addition, it is worthwhile to note that the observed "red" emission wavelengths coincide with the optical absorption range of the metallic-SWCNTs and infused PAH oligomers (Fig. 5(a)), and the resonance Raman conditions of PAH@metallic-SWCNTs (Fig. 6(a)). We begin with discussion about a possible formation of J-type aggregations because PAH oligomers are infused inside the very constrained volume of SWCNT spaces. Indeed, some dye molecules form the Jtype aggregates inside SWCNTs (e.g., $\alpha$-sexithiophene [34]). In our case, we can rule out the formation of J-aggregates due to the fact that the Raman enhancement is obscured 
by the $532 \mathrm{~nm}$ excitation, which energy is close to the optical bands of infused PAH oligomers.

In order to elucidate the red emission from the condensed sheet form, two mechanisms should be took into account. (1) The red emission simply originates from infused PAH oligomers and (2) exciplex emissions due to the mixed $\Pi^{-c o n j u g a t e d ~ s t a t e s ~ b e t w e e n ~}$ infused PAH oligomers and SWCNTs [35,36]. The former case (1) could occur when PAH oligomers are infused inside the semiconducting-SWCNTs. Since the semiconductingSWCNTs do not show optical absorption in the wavelength range of $600-800 \mathrm{~nm}$ (Fig. 5(a)), we can expect the red emission originating from the infused PAH oligomers, which elude absorption by the surrounding semiconducting-SWCNTs. We also consider the latter case (2) because the energy level of the infused PAH oligomers are close to the $\mathrm{M}_{11}$ transition of metallic-SWCNTs [37,38]. According to Itkis et al. [39], non-armchair metallic-SWCNTs inherently possess a very small energy gap induced by the curvature of the graphene sheet. Thus the charge recombination through fast relaxation at the Fermi level could be supressed. Here we can interpret the red-emission of PAH@SWCNT sheets due to the photo-induced charge transfer between the excited PAH oligomers and the metallic-SWCNTs. As we see in the resonance Raman analysis, electrons could be transferred from the metallic-SWCNTs to infused PAH oligomers or vice versa. Those 
results lead us to believe that excited electrons of both non-armchair metallic-SWCNTs and infused PAH oligomers can return to the ground state of the mixed $\Pi^{-}$conjugated states between infused PAH oligomers and the SWCNTs and finally emitting the red light.

\section{Conclusion}

We have demonstrated that pyrene and/or azupyrene oligomers infused SWCNTs exhibit the red fluorescence in a condensed sheet form, which is, in the most cases, quenched by the presence of metallic-SWCNTs and/or the charge transfer interaction with surrounding SWCNTs. Formation of the mixed $\Pi^{-}$conjugated state with metallicSWCNTs and infused PAH oligomers was revealed. The $\pi^{-c o n j u g a t e d ~ h y b r i d i z a t i o n ~}$ method reported here will open the way towards further developments of optoelectronic devices based on SWCNT nanohybrids without separating metallic- and semiconductingSWCNTs.

\section{Acknowledgement}

This work was supported by Exotic Nanocarbons, Japan Regional Innovation Strategy

Program by the Excellence, JST. T.F. was in part supported by JSPS KAKENHI Grant Numbers 25820331 and $15 \mathrm{H} 05544$. The synchrotron radiation experiments were 
performed at the BL02B2 of SPring-8 with the approval of the Japan Synchrotron Radiation Research Institute (JASRI) (Proposal No. 2013B1265).

\section{References}

[1] T. Mori, Y. Yamauchi, S. Honda, H. Maki, Nano Lett. 14 (2014) 3277.

[2] S. Y. Set, H. Yaguchi, Y. Tanaka, M. Jablonski, IEEE J. Sel. Top. Quant. Electron. $10(2004) 137$.

[3] M. E. Itkis, A. Yu, R. C. Haddon, Nano Lett. 8 (2008) 2224.

[4] K. Yanagi, R. Moriya, Y. Yomogida, T. Takenobu, Y. Naitoh, T. Ishida, H. Kataura, K. Matsuda, Y. Maniwa, Adv. Mater. 23 (2011) 2811.

[5] L. Cognet, D. A. Tsyboulski, J.-D. R. Rocha, C. D. Doyle, J. M. Tour, R. B. Weisman, Science 316 (2007) 1465.

[6] V. A. Karachevtsev, A. Yu. Glamazda, V. S. Leontiev, O. S. Lytvyn, U. DettlaffWeglikowska, Chem. Phys. Lett. 435 (2007) 104.

[7] J. G. Duque, L. Cognet, A. N. G. Parra-Vasquez, N. Nicholas, H. K. Schmidt, M. Pasquali, J. Am. Chem. Soc. 130 (2008) 2626.

[8] P. Cherukuri, S. M. Bachilo, S. H. Litovsky, R. B. Weisman, J. Am. Chem. Soc. $126(2004) 15638$. 
[9] S. M. Bachilo, M. S. Strano, C. Kittrell, R. H. Hauge, R. E. Smalley, R. B. Weisman, Science 298 (2002) 2361.

[10] M. J. O’Connell, S. M. Bachilo, C. B. Huffman, V. C. Moore, M. S. Strano, E. H. Haroz, K. L. Rialon, P. J. Boul, W. H. Noon, C. Kittrell, J. Ma, R. H. Hauge, R. B. Weisman, R. E. Smalley, Science 297 (2002) 593.

[11] T. Koyama, Y. Miyata, Y. Asada, H. Shinohara, H. Kataura, A. Nakamura, J. Phys. Chem. Lett 1 (2010) 3243.

[12] G.-H. Jeong, R. Hatakeyama, T. Hirata, K. Tohji, K. Motomiya, T. Yaguchi, Y. Kawazoe, Chem. Commun. 152 (2003).

[13] R. Kitaura, R. Nakanishi, T. Saito, H. Yoshikawa, K. Awaga, H. Shinohara, Angew. Chem. Int. Ed. 48 (2009) 8298.

[14] K. Yanagi, K. Iakoubovskii, H. Matsui, H. Matsuzaki, H. Okamoto, Y. Miyata, Y. Maniwa, S. Kazaoui, N. Minami, H. Kataura, J. Am. Chem. Soc. 129 (2007) 4992.

[15] T. Fujimori, A. Morelos-Gomez, Z. Zhu, H. Muramatsu, R. Futamura, K. Urita, M. Terrones, T. Hayashi, M. Endo, S.Y. Hong, Y.C. Choi, D. Tománek, K. Kaneko, Nat. Commun. 4 (2013) 2162. 
[16] T. Okazaki, Y. Iizumi, S. Okubo, H. Kataura, Z. Liu, K. Suenaga, Y. Tahara, M. Yudasaka, S. Okada, S. Iijima, Angew. Chem. Int. Ed. 50 (2011) 4853.

[17] A. I. Chernov, P. V. Fedotov, A. V. Talyzin, I. S. Lopez, I. V. Anoshkin, A. G. (2013) Nasibulin, E. I. Kauppinen, E. D. Obraztsova, ACS Nano 7 (2013) 6346.

[18] M. A. Loi, J. Gao, F. Cordella, P. Blondeau, E. Menna, B. Bártová, C. Hébert, S.

Lazar, G. A. Botton, M. Milko, C. Ambrosch-Draxl, Adv. Mater. 22 (2010) 1635.

[19] A. V. Talyzin, S. M. Luzan, K. Leifer, S. Akhtar, J. Fetzer, F. Cataldo, Y. O. Tsybin, C. W. Tai, A. Dzwilewski, E. Moons, J. Phys. Chem. C 115 (2011) 13207.

[20] A. V. Talyzin, I. V. Anoshkin, A. V. Krasheninnikov, R. M. Nieminen, A. G. Nasibulin, H. Jiang, E. I. Kauppinen, Nano Lett. 11 (2011) 4352.

[21] A. G. Anderson, Jr., A. A. MacDonald, A. F. Montana, J. Am. Chem. Soc., 1968, 90, 2993; A. G. Anderson, Jr., L. G. Kao, J. Org. Chem. 47 (1982) 3589.

[22] K. Yamazaki, N. Niitsu, K. Nakamura, M. Kanno, H. Kono, J. Phys. Chem. A $116(2012) 11441$.

[23] NIST Mass Spec Data Center, S. E. Stein, director, "Mass Spectra" in NIST Chemistry WebBook, NIST Standard Reference Database Number 69, Eds. P. J. Linstrom W. G. Mallard, National Institute of Standards and Technology, Gaithersburg MD, 20899, http://webbook .nist.gov, (retrieved February 3, 2016). 
[24] F. Banhart, J. Kotakoski, A. V. Krasheninnikov, ACS Nano 5 (2011) 26.

[25] A. Thess, R. Lee, P. Nikolaev, H. Dai, P. Petit, J. Robert, C. Xu, Y. H. Lee, S. G. Kim, A. G. Rinzler, D. T. Colbert, G. E. Scuseria, D. Tománek, J. E. Fischer, R. E. Smalley, Science 273 (1996) 483.

[26] M. Abe, H. Kataura, H. Kira, T. Kodama, S. Suzuki, Y. Achiba, K. Kato, M. Tanaka, A. Fujiwara, K. Matsuda, Y. Maniwa, Phys. Rev. B 68 (2003) 041405R.

[27] H. Kataura, Y. Kumazawa, Y. Maniwa, I. Umezu, S. Suzuki, Y. Ohtsuka, Y. Achiba, Synth. Met. 103 (1999) 2555.

[28] R. B. Weisman and S. M. Bachilo, Nano Lett. 3 (2003) 1235.

[29] M. J. Frisch, G. W. Trucks, H. B. Schlegel, G. E. Scuseria, M. A. Robb, J. R. Cheeseman, G. Scalmani, V. Barone, B. Mennucci, G. A. Petersson, H. Nakatsuji, M. Caricato, X. Li, H. P. Hratchian, A. F. Izmaylov, J. Bloino, G. Zheng, J. L. Sonnenberg, M. Hada, M. Ehara, K. Toyota, R. Fukuda, J. Hasegawa, M. Ishida, T. Nakajima, Y. Honda, O. Kitao, H. Nakai, T. Vreven, J. A. Montgomery, Jr., J. E. Peralta, F. Ogliaro, M. Bearpark, J. J. Heyd, E. Brothers, K. N. Kudin, V. N. Staroverov, R. Kobayashi, J. Normand, K. Raghavachari, A. Rendell, J. C. Burant, S. S. Iyengar, J. Tomasi, M. Cossi, N. Rega, J. M. Millam, M. Klene, J. E. Knox, J. B. Cross, V. Bakken, C. Adamo, J. Jaramillo, R. Gomperts, R. E. Stratmann, O. Yazyev, A. J. Austin, R. Cammi, C. 
Pomelli, J. W. Ochterski, R. L. Martin, K. Morokuma, V. G. Zakrzewski, G. A. Voth, P. Salvador, J. J. Dannenberg, S. Dapprich, A. D. Daniels, O. Farkas, J. B. Foresman, J. V. Ortiz, J. Cioslowski, D. J. Fox, Gaussian09, Gaussian, Inc., Wallingford CT, 2009. [30] A. Jorio, M. A. Pimenta, A. G. Souza Filho, R. Saito, G. Dresselhaus, M. S. Dresselhaus, New J. Phys. 5 (2003) 139.

[31] A. Campion, P. Kambhampati, Chem. Soc. Rev. 27 (1998) 241.

[32] M. R. Kagan, R. L. McCreery, Anal. Chem. 66 (1994) 4159.

[33] S. Huang, X. Ling, L. Liang, Y. Song, W. Fang, J. Zhang, J. Kong, V. Meunier, M. S. Dresselhaus, Nano Lett. 15 (2015) 2892.

[34] E. Gaufrés, N. Y.-Wa Tang, F. Lapointe, J. Cabana, M.-A. Nadon, N. Cottenye, F. Raymond, T. Szkopek, R. Martel, Nat. Photonics 8 (2014) 72.

[35] N. Mackiewicz, J. A. Delaire, A. W. Rutherford, E. Doris, C. Mioskowski, Chem. Eur. J. 15 (2009) 3882.

[36] Y. Sun, S. R. Wilson, D. I. Schuster, J. Am. Chem. Soc. 123 (2001) 5348.

[37] M. Tange T. Okazaki, S. Iijima, J. Am. Chem. Soc. 133 (2011) 11908.

[38] N. Matsumoto, M. Nishiyama, C. Adachi, J. Phys. Chem. C 112 (2008) 7735.

[39] M. E. Itkis, S. Niyogi, M. E. Meng, M. A. Hamon, H. Hu, R. C. Haddon, Nano Lett. $2(2002) 155$. 


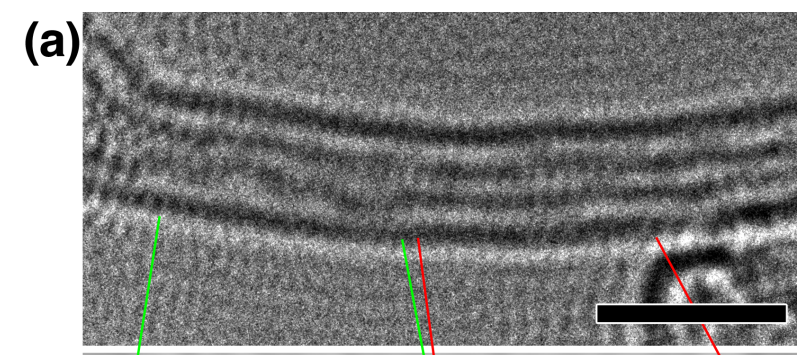

(b)

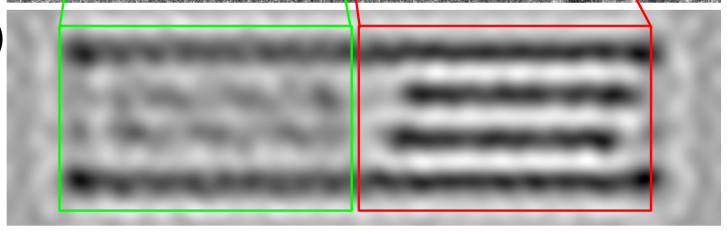

(c)

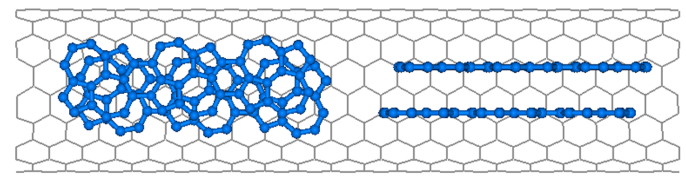

(d)

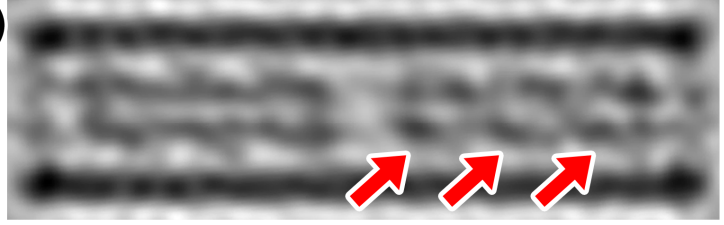

(e)

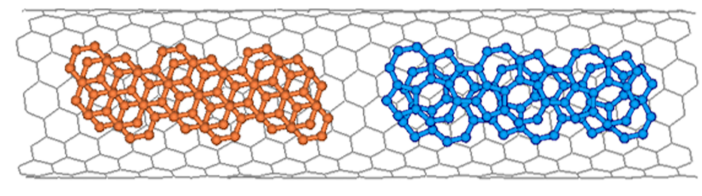

Figure 1. (color online) (a) A HRTEM image of PAH oligomers infused inside an SWCNT.

Scale bar: $2 \mathrm{~nm}$. (b) A simulated image of the model structure shown in (c). (c) The model structure of azupyrene oligomers inside $(9,9)$ SWCNT. Molecular plane is set perpendicular (left) and parallel (right) against the incident electron beam. (d) A simulated image of the model structures shown in (e). Red arrows indicate the characteristic simulated image of azupyrene oligomers inside the SWCNT. (e) The model structures of pyrene oligomers (left) and azupyrene oligomers (right) infused inside $(11,7)$ SWCNT. 
(a)

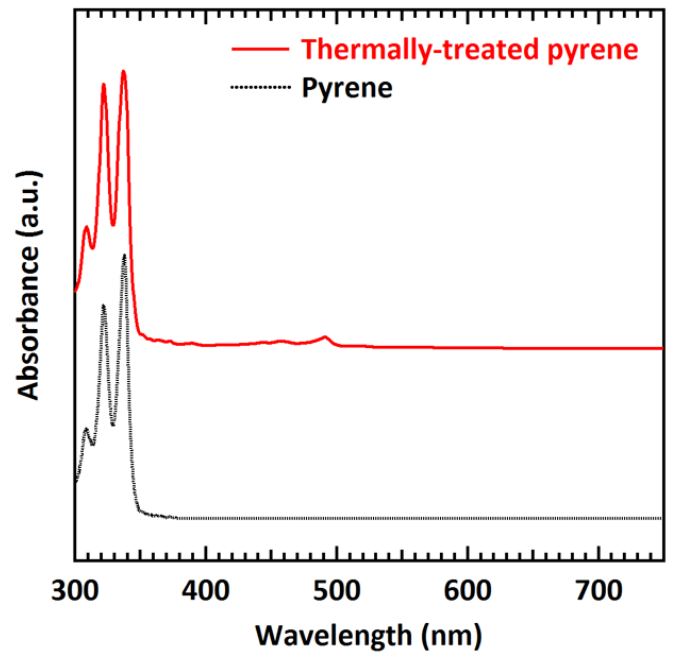

(b)

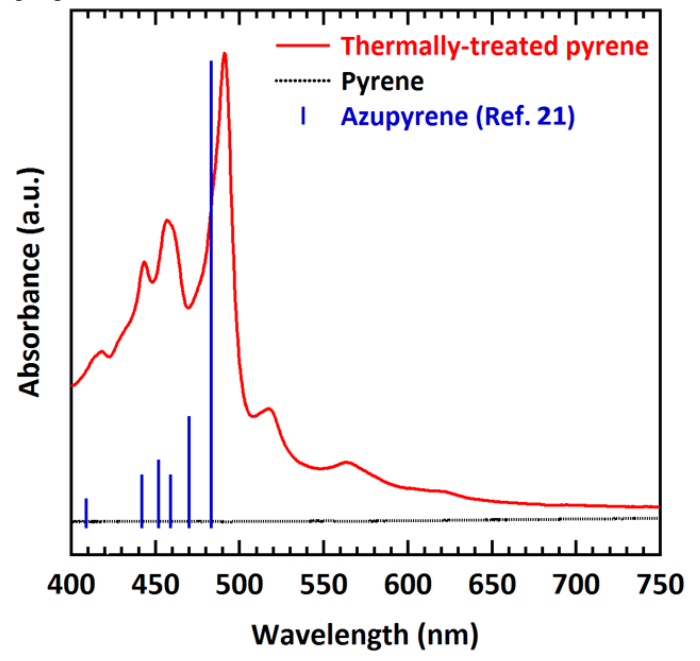

Figure 2. (color online) (a) Optical absorption spectra of thermally-treated pyrene (solid

line) and pyrene (dotted line) in toluene solutions. (b) Magnified spectra of (a) in the wavelength range of $400-750 \mathrm{~nm}$. Bars indicate the reference values of azupyrene [21]. 
(a)

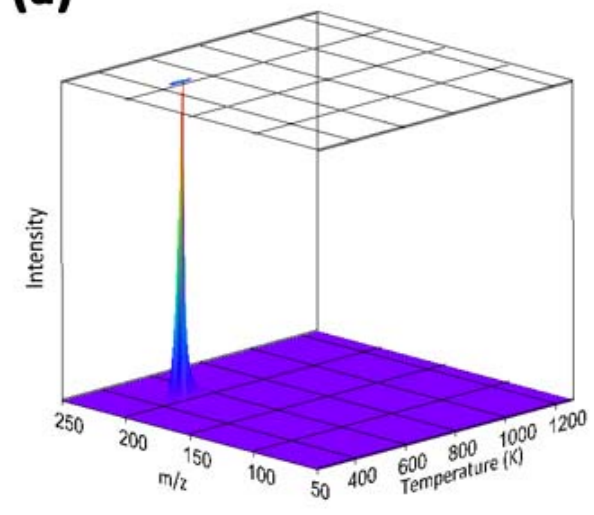

(b)

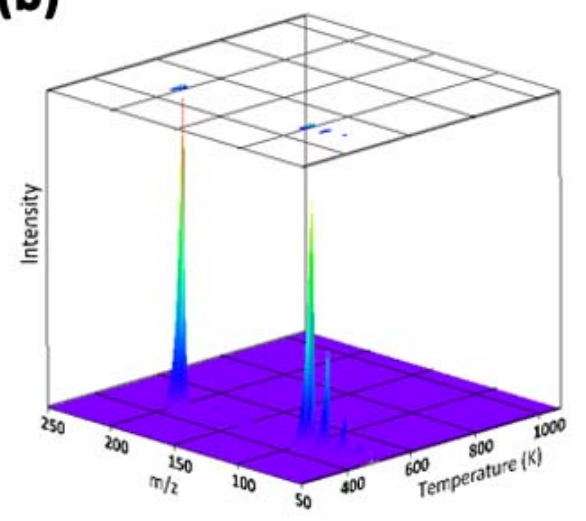

(c)

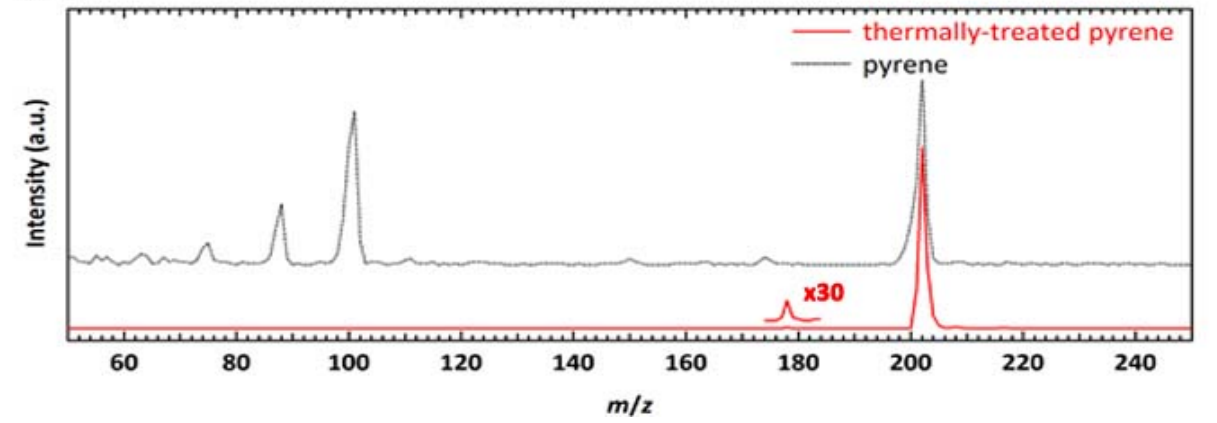

Figure 3. (color online) TG-mass spectra of (a) thermally-treated pyrene and (b) pyrene.

(c) Mass spectra of thermally-treated pyrene (solid line) and pyrene (dotted line) obtained at $T=480 \mathrm{~K}$ in (a) and (b). 


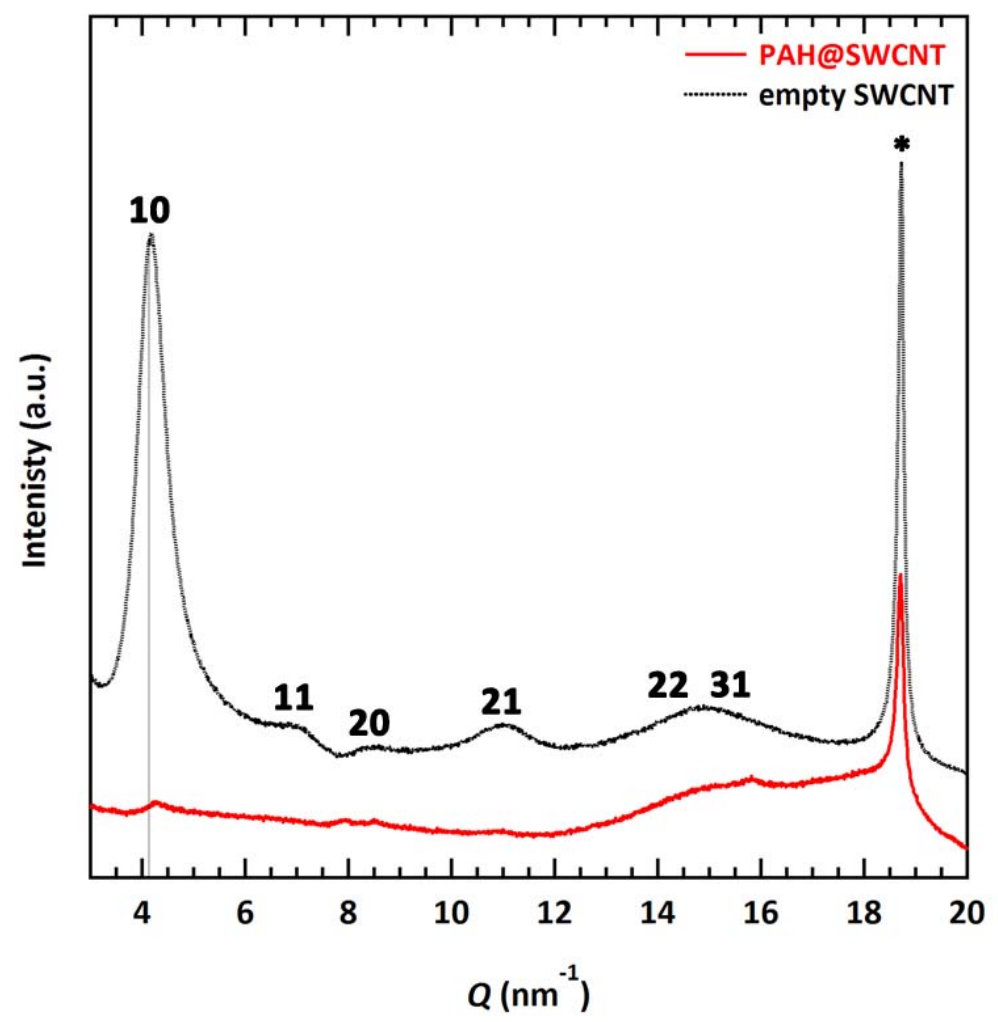

Figure 4. (color online) XRD profiles of empty SWCNTs (dotted line) and PAH@SWCNTs

(solid line). Asterisk indicates the (002) peak of impurity graphite. 

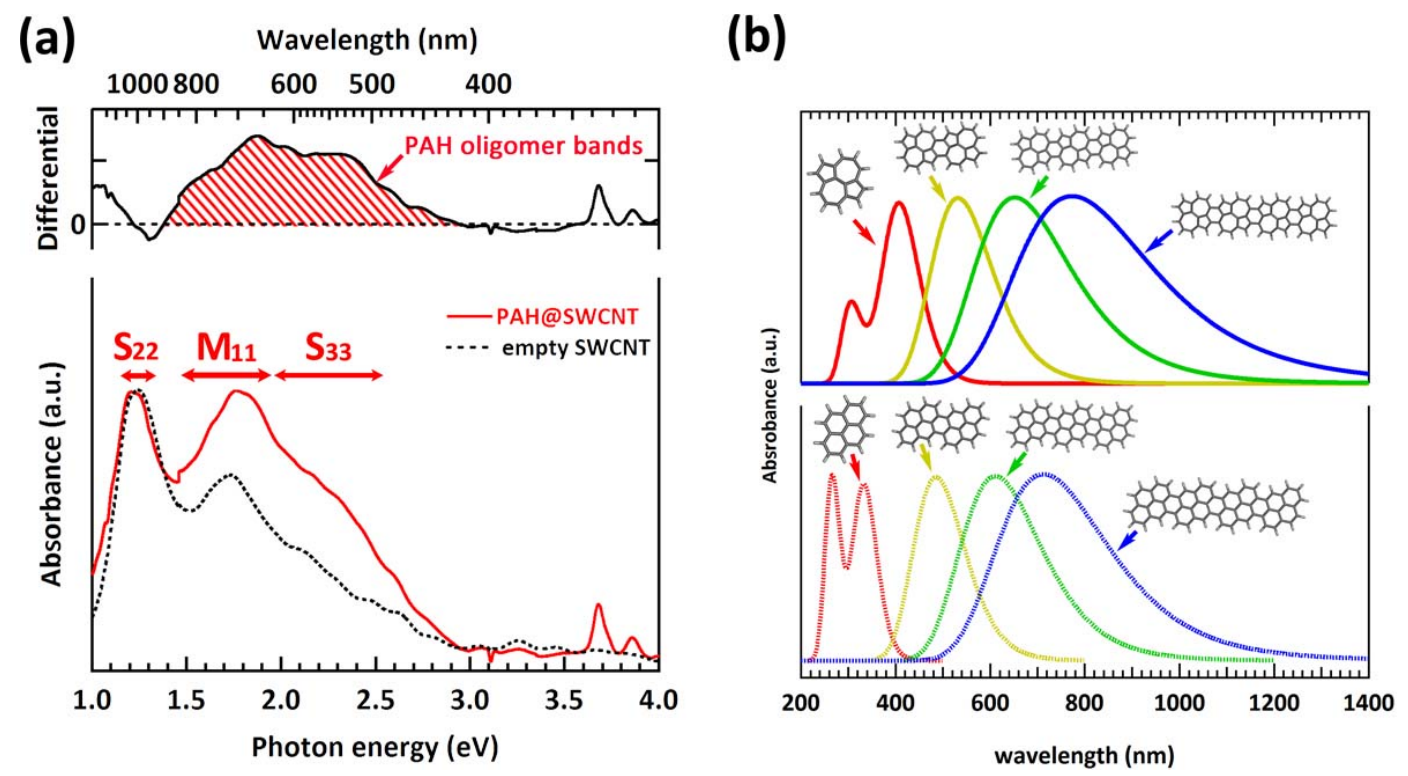

Figure 5. (color online) (a) Optical absorption spectra of empty SWCNT (black dotted line) and PAH@SWCNT (red solid line) in 1 wt\% aqueous SDS solution (lower figure).

The differential spectrum of empty SWCNT and PAH@SWCNT is shown in the upper plane (black solid line). $\mathrm{M}_{11}, \mathrm{~S}_{22}$, and $\mathrm{S}_{33}$ indicate the first electronic transition bands of metallic-SWCNTs, the second- and third-narrowest electronic transition bands of semiconducting-SWCNTs, respectively [27]. (b) TD-DFT calculated optical absorption spectra of azupyrene monomer and its oligomers (solid lines in the upper figure) and those of pyrene monomer and its oligomers (dotted lines in the lower figure). 

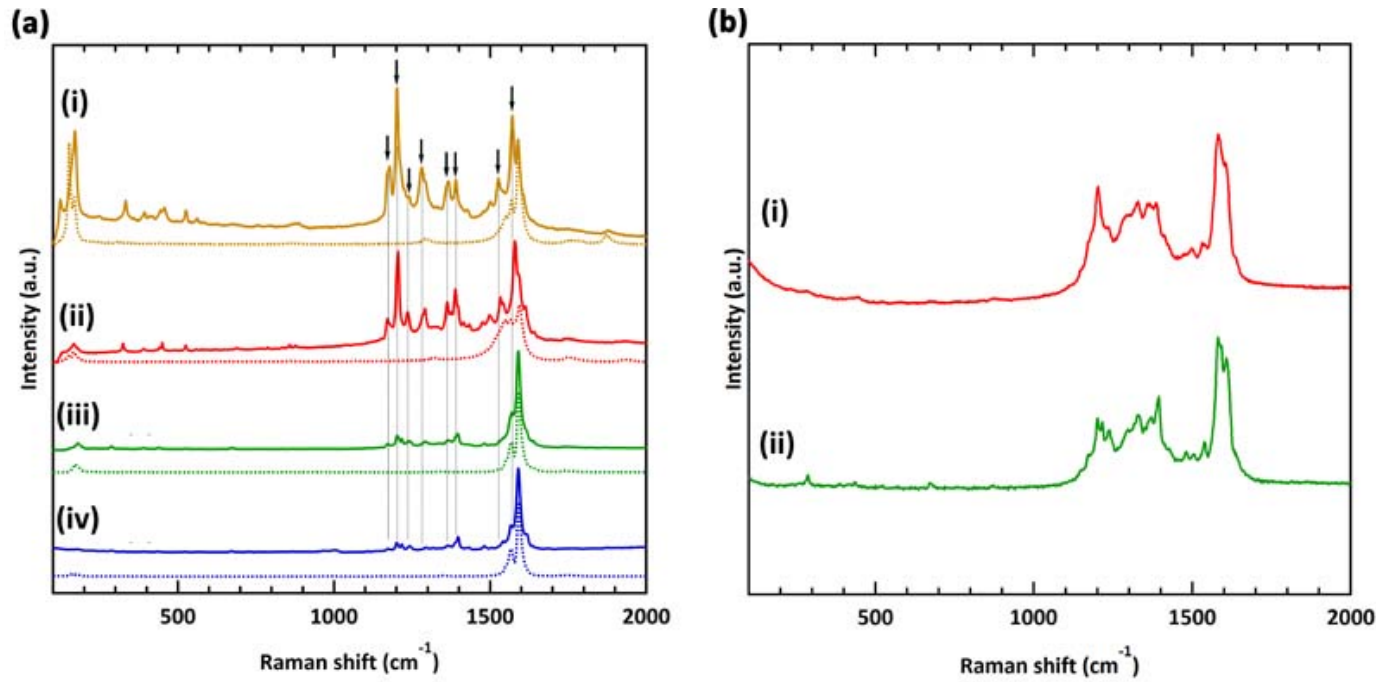

Figure 6. (color online) (a) Resonance Raman spectra of PAH@SWCNTs (solid lines) and empty SWCNTs (dotted lines) obtained with (i) 785, (ii) 633, (iii) 532, and (iv) $488 \mathrm{~nm}$ laser excitations. Characteristic Raman peaks of infused PAH oligomers are highlighted by arrows. (b) Raman spectra of thermally-treated pyrene obtained with (i) 633 and (ii) $532 \mathrm{~nm}$ laser excitations. 

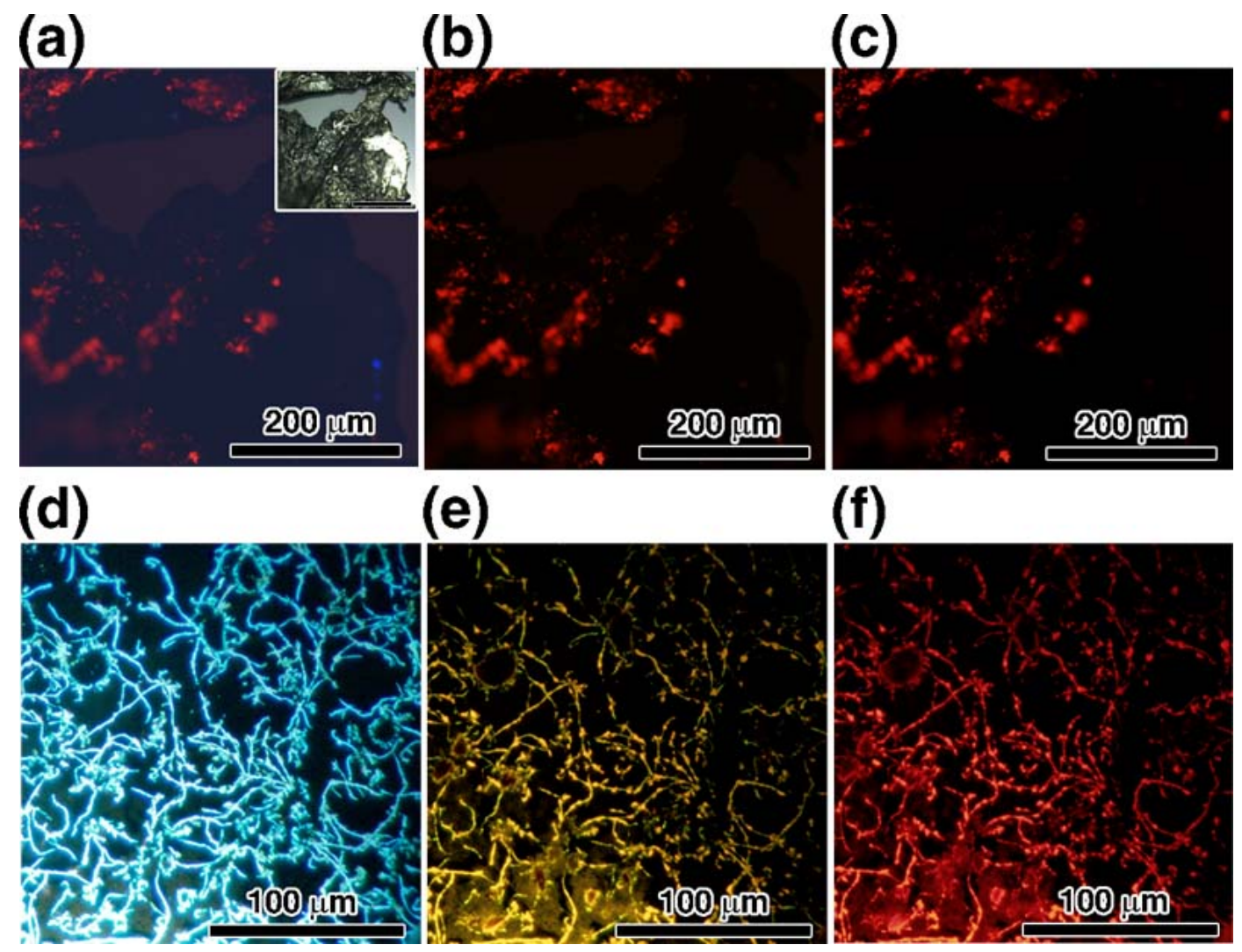

(f)

Figure 7. (color online) Fluorescent microscopic images of (a-c) PAH@SWCNT sheet and

(d-f) thermally-treated azupyrene excited with $\lambda_{\mathrm{ex}}=360-370 \mathrm{~nm}$ (left), $470-495 \mathrm{~nm}$

(middle), and $530-550 \mathrm{~nm}$ (right) light sources. Inset in (a) shows an optical microscope image of PAH@SWCNT sheet observed at the same images in (a-c). Detection wavelengths are $>420 \mathrm{~nm},>510 \mathrm{~nm}$, and $>575 \mathrm{~nm}$ for ultraviolet, blue, and green light excitations, respectively. 


\section{Graphical Abstract}

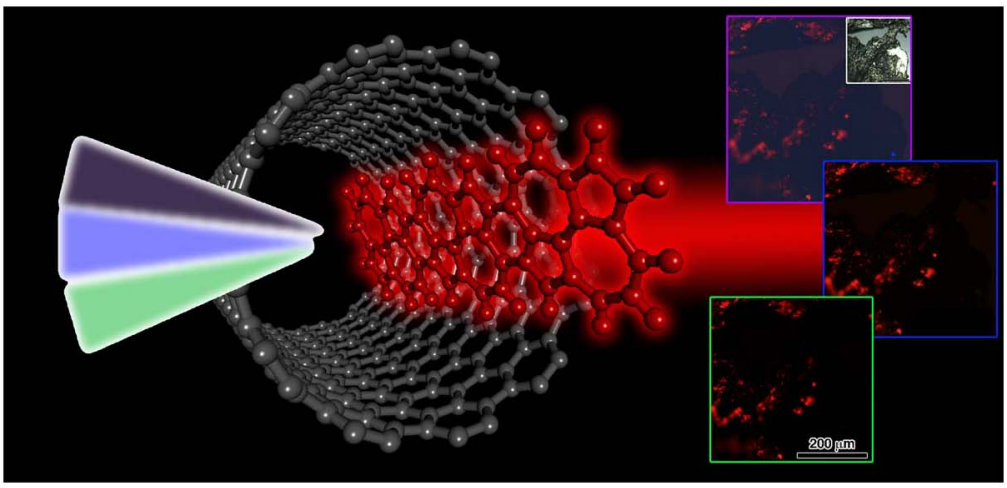

